Aus der chirurgischen Abteilung des städtischen Krankenhauses zu Hildesheim (Direktor: Medizinalrat Dr. Becker).

\title{
Über das familiäre Auftreten von Darmpolypen.
}

Von Dr. Walter Thorbecke, ehem. Assistenzarzt.

(Mit I Abbildung.)

Das Krankheitsbild der Polyposis intestini war bis in die Mitte des vorigen Jahrhunderts noch so gut wie unbekannt. Die erste Beschreibung der Polyadenomata tractus intestinalis findet sich in der von Sklifasowski im Jahre I88I veröffentlichten gleichnamigen Monographie. Während um diese Zeit I4 von Port beschriebene - Fälle bekannt sind, gibt Döring I 907 in einer Abhandlung „Die Polyposis intestini und ihre Beziehung zur carcinomatösen Degeneration" (Langenbecks Archiv für klinische Chirurgie) eine tabellarische Zusammenstellung über 50 Fälle.

Diese statistische Übersicht hat Versé in seinen ausführlichen Untersuchungen „Über die Entstehung, den Bau und das Wachstum der Polypen, Adenome und Carcinome des Magenund Darmkanals" mit einigen Änderungen aufgenommen und etwas erweitert. Im übrigen bilden den Hauptinhalt dieser Arbeit Untersuchungen von Polypen und Carcinomen des Magen-Darmkanals mit anschließenden epikritischen Erörterungen und histogenetischen Schlußfolgerungen. Während Döring die Polyposis mehr auf ihre klinischen Erscheinungen hin untersucht.

Von Arbeiten aus der neueren Zeit, die sich näher auch mit der Polyposis und im Anschluß daran mit histogenetischen Fragen beschäftigen, möchte ich besonders die von Wechselmann „Polyp und Carcinom im Magen-Darmkanal“" (Beịträge zur klinischen Chirurgie I9Io, Bd. 70) erwähnen. Ferner Ribber $\mathrm{t}$ „Darmpolyp und Carcinom“ (Frankfurter Zeitschrift für Pathologie 1909, Bd. 2, Heft 4) und daselbst „Das Carcinom des Menschen" I9II, Bonn. 
Ribbert führt in der Literaturübersicht noch 8 Arbeiten über Polyposisfälle an, die für diese Frage bis jetzt noch nicht verwertet sind. Von diesen habe ich 6 , die mir zugänglich geworden sind, kurz beschrieben und der Tabelle eingeordnet. Dazu kommen die seit 1908 veröffentlichten und mir bekannt gewordenen, zum Schluß die hier im städtischen Krankenhause beobachteten 3 Polyposiserkrankungen. Ich möchte im folgenden zunächst eine kurze Darstellung dieser, seit der letzten Veröffentlichung von Versé bekannt gewordenen Fälle geben.

I. Charles A. Morton.

45jährige Frau. Die Dickdarmschleimhaut ist mit über Ioo Polypen besetzt, besonders im unteren Teil des Colon, der Flexura sig. moidea und dem Rektum. Es finden sich zwei typische, stark ul. cerierte Adenocarcinome. Das eine dicht unter der Flexur sitzende hat die Wand infiltriert und verengt das Lumen so, daß gerade der kleine Finger noch durchgeht. Die unteren 2-3 Zoll des Rektums waren I4 Tage vor dem Tode exstirpiert. Es besteht ein Krebs von adenomatösem Charakter, teils schwammig, teils ulceriert. Zwischen diesem krebsartigen Gewächse und dem andern in der Sigmoidealflexur ist die Schleimhaut mit Polypen besetzt.

II. Q uén u und Landel.

Ein 6zjähriger Mann, dessen Vorfahren gesund sind, ist seit vier Monaten an hartnäckigen, aller Behandlung trotzenden Durchfällen erkrankt. Operation. Rektumexstirpation von $38 \mathrm{~cm}$ Länge. Die Schleimhaut ist mit Polypen bedeckt. Es findet sich ein 'Tumor an der rechten Seite des Beckenanteils des Colons, und weiter unten ein Rektumkrebs. Der Kranke stirbt am 14. Tage nach der Operation an Bronchopneumonie.

III. R obs on - Knaggs.

27jähriger Mann hat vor sechs Jahren an typhoidem Fieber gelitten, ist seit einem Jahr an Hämorrhoiden erkrankt. Seit 4 Monaten Diarrhöen nud Gewichtsabnahme. Dauernde Tenesmus und Schmerzen in der Sakralgegend. Kolostomie. 3 Monate danach Exitus. Bei der Sektion zeigt sich der Pylorus durch einen größeren Polypen verschlossen. Am Anus und Rektum je ein ulcerierter Tumor. Ebenso einer unter der Kolostomiewunde. Die Schleimhaut zwischen den Krebsen ist polypös.

IV. Stein thal.

50jährige Frau. I899 Entfernung eines hühnereigroßen polypösen Rektumtumors. F eststellung von Exkreszenzen in der Umgebung des Tumors. 1906 wegen Rezidivierung Abtragung eines $18 \mathrm{~cm}$ langen Rektumstücks. Wucherung auf das Rektum beschränkt. Zurzeit Heilung. 
V. Steinthal.

3Ijährige Frau. Rektumcarcinom. Viele Polypen (gutartige Adenome).

VI. W e chselmann.

Außer der Angabe, daß der 43jährige Patient an Ileuserscheinungen zugrunde gegangen ist, findet sich nur pathologischer Befund. Im Colon descendens cine große Menge hirsekorn- bis erbsengroße, zum Teil langgestielte, zum Teil breitbasig aufsitzende Polypen; ebenso im Anfangsteil des Rektums und in der Ampulle. Je ein Tumor an der Flexura hepatica, mitten im Colon transversum, ferner $12 \mathrm{~cm}$ oberhalb der Analöffnung und in der Analöffnung selbst (zirkulär). Die Tumoren sind in die Muscularis hineinwachsend, von teils drüsenartigem Zellaufbau, teils von alveolär massivem Charakter. - Nachweis beginnender carcinomatöser Entartung in Polypen.

VII. W e chs elman $n$.

Im ganzen Dickdarm des igjährigen Mannes finden sich zahlreiche Polypen bis zu 6 und $8 \mathrm{~cm}$ Länge. Handbreit oberhalb des Anus ein ulcericrtes ringförmiges Carcinom; oberhalb und unterhalb davon sind die Polypen stark gehäuft. Beschreibung von Carcinommetastasen, die von der Basis her in den Polypen hinein wuchern. Beobachtung von zwei Carcinomanlagen an einem Polypen, nämlich ciner primären, von der Spitze eindringenden und einer metastatisch sekundären, die sich von unten nach oben verbreitet.

VIII. Wechselman $\mathrm{n}$.

Es handelt sich um einen 63jährigen Mann, der an einer doppelseitigen Bronchopneumonie gestorben ist. Die Verteilung der Polypen auf den ganzen Darmtraktus ist folgende: Magen und Duodenum je ein Tumor, Jejunum 3, Colon I 40. Massenhaftes Auftreten unterhalb der Valvula im Cöcum und im Rektum und an den Flexuren. Breitbasiges oder dünngestieltes Aufsitzen mit glatter, teils höckeriger Oberfläche. Der mikroskopische Befund ergibt in allen Tumoren Kombination von Epithelien und Bindegewebswucherung.

IX, B a bler, Nibrügge, I' is ch.

I9jähriges Mädchen leidet seit 2 Jahren an anfangs spärlichen und nur zeitweise mit Blut untermischten, später gehäuften und dauernd blutigen Durchfällen. Da das Leiden für chronischen Dickdarmkatarrh gehalten wurde, Einnähung des Wurmfortsatzes in die Haut und Spülung. Trotz anfänglicher Besserung Tod nach 9 Tagen. Heredität? Sektionsbefund: Dickdarmschleimhaut vom Blinddarm bis zur Flexur sehr verdickt, dicht mit polypösen Wucherungen besetzt. Adenocarcinom zwischen Muscularis und Mucosa in der ganzen beschricbenen Ausdehnung.

$\mathrm{X}$. Oseki.

3rjährige Frau stirbt nach einjähriger Krankheit an CarcinomKachexie. Keine Heredität. Patientin hatte mit 22 Jahren einen 
Mastdarmvorfall, der sich später während einer Geburt und dann noch einmal beim Heben einer Last wiederholte. Seit dieser Zeit Abmagerung, Schmerzen in den Beinen und im Leibe. Sektionsbefund: Carcinoma recti exulceratum. Ployposis intestini crassi. Außerdem verschiedene Metastasen.

$X I$. Küt $\mathrm{tn}$ er.

33jähriger Mann leidet seit Io--12 Jahren an häufig mit Blut vermischten Durchfällen. In den letzten 6 Monaten Tenesmus und stärkere Blutung. Befund der digitalen bzw. rektoromanoskopischen Untersuchung: Rektumcarcinom der Vorderwand und typische Polyposis (stecknadelkopf- bis bohnengroße, zum Teil gestielte Polypen). Keine Heredität.

XII. Brentano.

42jähriges, gut genährtes Dienstmädchen, an blutig schleimigen Abgängen aus dem Mastdarm und schmerzhaften Tenesmen leidend. Heredität fraglich.

Befund: Ringförmige, weiche, zottige Geschwuist, die das Lumen verengert, nach oben nicht abgrenzbar ist.

Mastdarmresektion nach $\mathrm{Kr}$ a ske mit guter Heilung und Nachlassen der Beschwerden. Dic rektale Untersuchung ergibt jetzt Vorhandensein multipler Polypen. Mikroskopische Untersuchung der Drüsen ergibt Hypertrophie und Hyperplasie.

XIII. B rentano.

Schon seit dem 8. Lebensjahre ist bei dem 3ojährigen Manne ein Polyp bei jedem Stuhlgang vorgedrängt. Mikroskopisch finden sich einfache adenomatöse Polypen neben anderen, die typisch carcinomatöse Veränderungen aufweisen. Es handelt sich um ein ZylinderEpithel-Carcinom. Heredität?

XIV. A. Albu.

U. a. wird ausgeführt: „Bösartige Polypen sind häufiger als gutartige. Zweimal konnte bösartige Umwandlung eines ursprünglich benignen Polypen beobachtet werden."

2jähriges Mädchen ist an immer wiederkehrenden Darmblutungen erkrankt. Befund: $7 \mathrm{~cm}$ ab ano beginnende Polyposis.

XV. A. Alb u.

I6jähriges Mädchen, ebenfalls an Darmblutungen erkrankt. Befund: Polyposis der Ampulla recti.

XVI. S chneider.

„8jähriges Mädchen, seit etwa 4 Jahren krank, zeigt hochgradige Anämie. Odeme, kachektische und urämische Symptome als schwer verständliche Folgen einer Polyposis des unteren Darmabschnitts. Wie immer tödlicher Ausgang."

XVII. Helferich.

35 jähriger, sehr bla $B$ aussehender Mann, leidet seit $3 \frac{1}{2} / 2$ Jahren an anfallsweise auftretenden Durchfällen, die mit Verstopfung ab- 
wechseln. Seit den letzten Jahren ist jedem Stuhl Blut beigemischt. Keine Heredität. Befund: $6 \mathrm{~cm}$ oberhalb des Orificium Tumor von festweicher Konșistenz, nach oben nicht abgrenzbar. Anlegung eines Anus praeternaturalis. Die ganze Rektalschleimhaut ist mit erbsengroßen und größeren Polypen bedeckt. Keine maligne Entartung der Polypen.

XVIII. Esser.

Die Krankengeschichte betrifft einen 29jährigen Fabrikarbeiter mit ausgedehnter Polyposis. Anlegung eines Anus praeternaturalis. Besserung der Darmfunktionen.

XIX. Prag.

Fall von Polyposis des Colons, behandelt mit Appendicostomie und Spülungen des Colons mit I proz. Argentum nitricum-Lösung von der Fistelöffnung aus. Verfasser hält die Polypen für angeboren, die Reizerscheinungen, die gelegentlich auch fehlen können, für eine Folge.

$\mathrm{XX}$.

XXI. Eigene Beobachtungen.

XXII.

Erwähnen möchte ich noch :

Lynch.

Zwei Fälle von multipler Polyposis des Rektum und unteren Flexura sigmoidea. In dem einen kam es zum Exitus trotz ausreichender Ennährung. L ynch nimmt an, daß es sich dabei um eine carcinomatöse Entartung der Polypen gehandelt habe, wie denn eine solche in 40 Proz. der Fälle eintreten soll.

In der folgenden Übersicht habe ich die Tabelle Versés bzw. Dörings fortgeführt und durch die Rubrik „Krankheitssymptome" erweitert.

Dic 3 hier beobachteten Fälle betreffen die Brüder Wilhelm und Fritz G., sowie den 5 jährigen Sohn des letzteren. Alle drei, die äußerlich schon den nämlich krankhaft blassen elenden Eindruck machen, leiden in gleicher Weise an den klinischen Erscheinungen der Polyposis, nämlich schleimig-blutigen Durchfällen seit ihrer frühesten Kindheit. Das Krankheitsbild tritt uns als ganz ausgesprochen familiär entgegen.

Beide Brüder sind vor Jahren, ehe sie jetzt zur Behandlung bzw. Beobachtung kamen wegen hämorrhoidalen Beschwerden und Einklemmungserscheinungen infolge vorgefallener Polypen -und zwar Fritz G. zu wiederholten Malen - operiert worden, wobei 


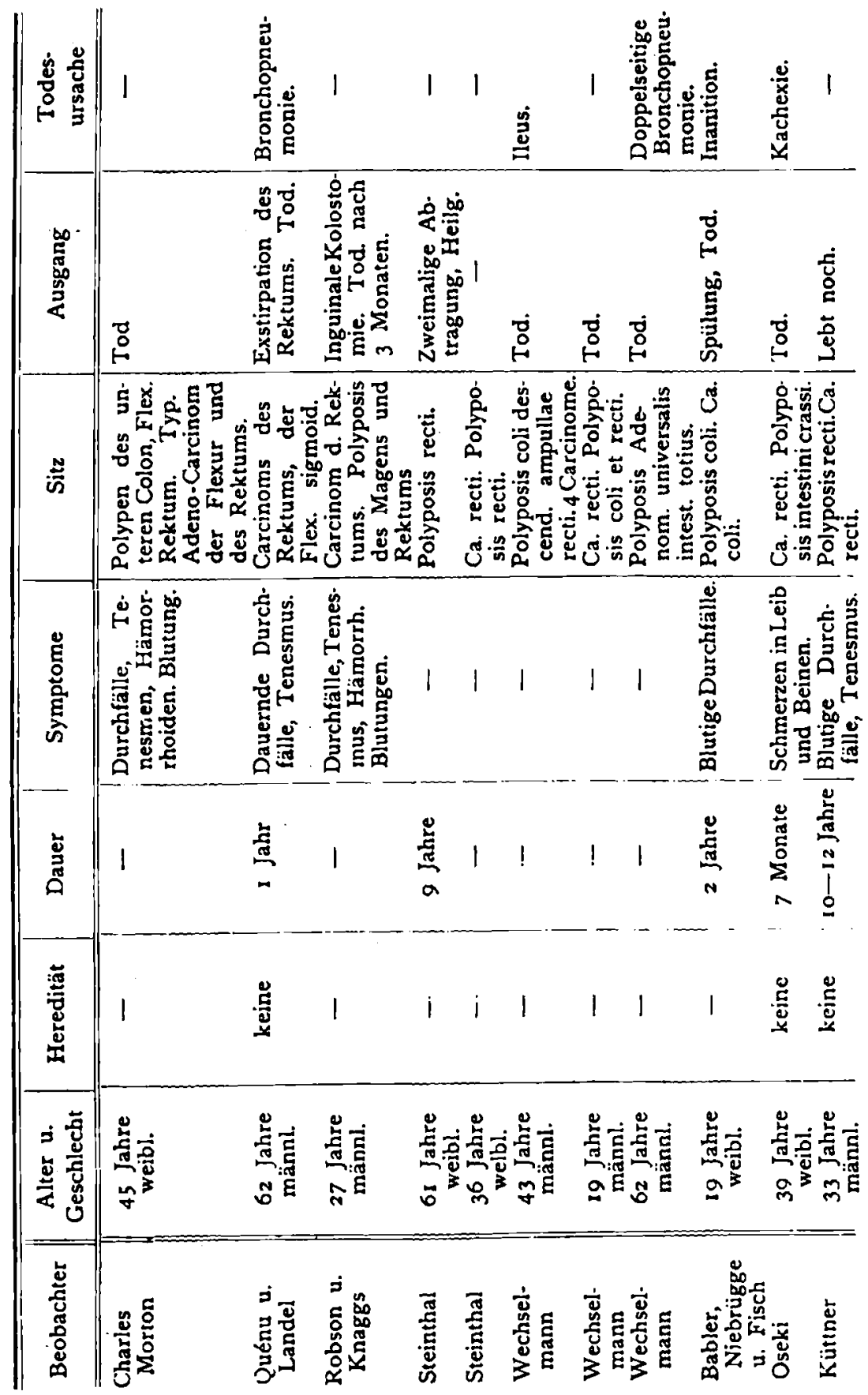




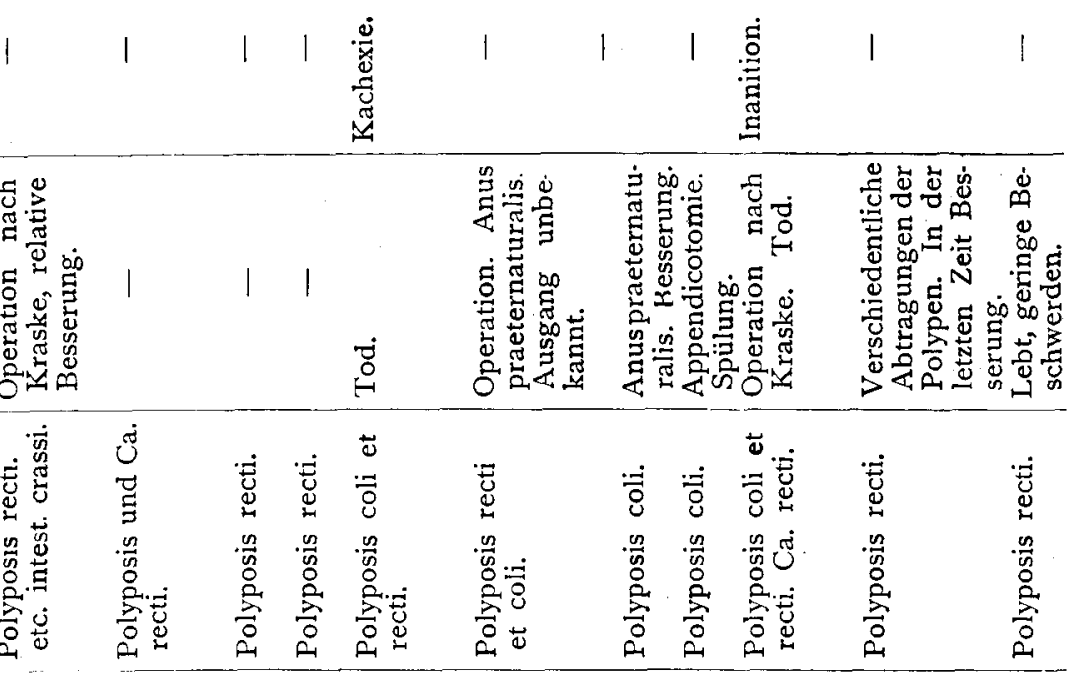

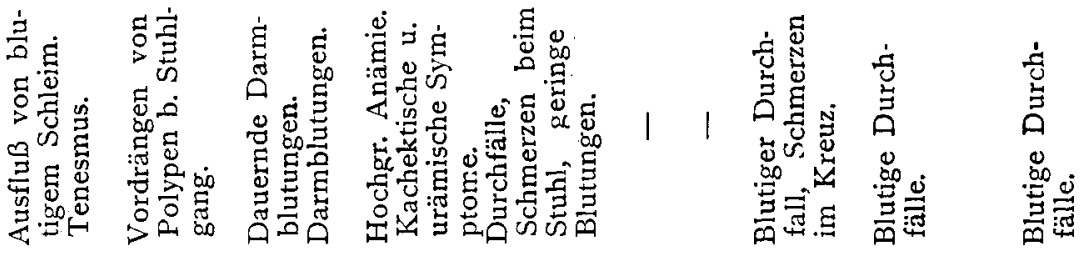

\begin{tabular}{|c|c|c|c|c|c|c|c|c|c|c|}
\hline 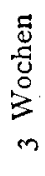 & 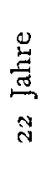 & 1 & 1 & $\underset{+}{\stackrel{\Xi}{\Xi}}$ & 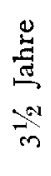 & 1 & 1 & 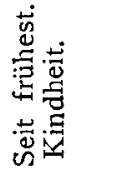 & $\dot{8}$ & 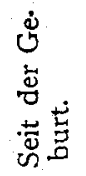 \\
\hline 1 & 1 & 1 & 1 & 1 & $\underset{ \pm}{\stackrel{ \pm}{ \pm}}$ & 1 & $!$ & 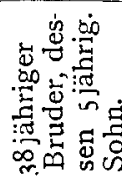 & 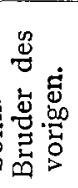 & 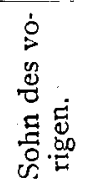 \\
\hline
\end{tabular}

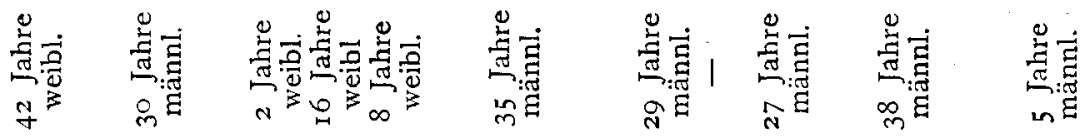

\begin{tabular}{|c|c|c|c|c|c|c|c|c|c|}
\hline 总 & 1 & $\stackrel{\Xi}{\varrho}$ & 党 & 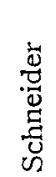 & 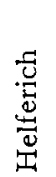 & $\begin{array}{l}\breve{0} \\
\text { जn } \\
\text { [n] }\end{array}$ & 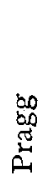 & 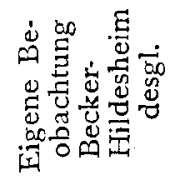 & $\begin{array}{l}\dot{0} \\
\dot{0} \\
\dot{0}\end{array}$ \\
\hline
\end{tabular}


die Diagnose Polyposis gestellt wurde. Wilhelm G. fand dann wegen seit Pfingsten verstärkt auftretender Rücken- und Steißbeinschmerzen im Oktober vorigen Jahres erneut hier Aufnahme. Er wurde an Rectumcarcinom operiert nach Kraske - trotz hochgradiger Schwäche mit günstigem Ausgang. Nach' einem halben Jahre erfolgte der Tod an Inanition. Die Sektion ergab Polyposis coli et recti ohne Metastasen. Mikroskopisch erwies sich der Krebs als Adenocarcinom und die Polypen als einfache Adenome. Im einzelnen läßt sich über die drei Fälle folgendes sagen :

Fall I. Wilh elm G. wurde im Jahre 1908 zum erstenmal wegen Darmpolypen im Krankenhause aufgenommen. Die damalige Krankengeschichte soll zunächst unverändert und unverkürzt wiedergegeben werden.

Wilhelm G. 23. XI. bis 9. XII. 1908.

Multiple Papylome des Rektums.

A n a mnese: Ein Bruder soll Hämorrhoiden haben. Ist hier vor $6-7$ Jahren operiert worden. Als Kind einmal im Sommer in Salzdetfurt. Nicht Soldat, weil zu schwach. Seit etwa einem Jahre bemerkt Patient, daß beim Stuhlgang Blutungen auftreten, die anfangs weniger reichlich waren; in der letzten Zeit wurden sie stärker. Die Hämorrhoidalknoten gingen nicht von selbst zurück, sondern mußten stets manuell reponiert werden. Oft traten dabei Blutungen auf, die in der letzten Zeit stärker wurden. Dr. W. schickt Patient zur Operation.

B ef und: Blaß aussehender, junger Mann in mittlerem Ernährungszustand. Innere Organe o. B. Am After sieht man äußerlich nur einen unbedeutenden Hämorrhoidalknoten. Beim Touschieren findet man eine Anzahl flottierender weicher Tumoren. Es wird dem Patienten ein Einlauf gemacht und er aufgefordert, in hockender Stellung zu pressen. Dabei tritt eine etwa faustgroße Masse heraus, die fast den Eindruck einer Blasenmole macht. Sie besteht aus einer großen Anzahl erbsen- bis walnußgroßer, weicher, teilweise gestielter Polypen, die sehr bluten. Den Ansatz der Massen kann man nicht deutlich erkennen.

25. XI. Operation in Lumbalanästhesie (Tropococain 0,075 , Injektion).

Nachdem durch vorheriges Pressen die Tumoren herausgetreten sind, werden die einzelnen Partien mit der Faßzange abgeklemmt und mit Katgut unterbunden. Nachdem so der größte Teil abgetragen ist, sieht man, daß die hintere Rektumwand nicht weit oberhalb des Sphincter externus den Ausgangspunkt der Hauptmasse bildet. Daneben aber findet sich, wie durch Inspektion mittels Speculum fest- 
gestellt wird, im ganzen Bereiche des Rektums bis über den Sphincter tertius hinaus noch eine größere Anzahl meist gestielter, teilweise bohnengroßer Polypen, die in der Mehrzahl allein sitzen, nicht wie die Hauptmasse - ein Konglomerat bilden. Die erreichbaren größeren Polypen werden entfernt. Es bleibt noch immer eine Menge kleinerer zurück, die sich radikal nur durch die Resektion des Rektums würden entfernen lassen. Bei der offenbaren Gutartigkeit wird davon abgesehen. Tamponade des Rektums mit Vioformgaze. Opium, Bettruhe.

Im weiteren Verlauf der Heilung werden bei dem Patienten wiederholt blutige Stühle festgestellt. Im übrigen schnelle Erholung. Gewicht am 23. XI. $58 \mathrm{~kg}$, 3. XII. $57 \mathrm{~kg}$, 9. XII. $59 \mathrm{~kg}$. Entlassen mit der Weisung, wieder zu kommen, wenn die stehen gebliebenen Papillome Beschwerden verursachen. Arbeitsfähig.

Vorgeschichte: Die jetzige Aufnahme des Goertz erfolgt am 2I. X. 19I2. Er klagt hauptsächlich über unerträgliche Schmerzen im Rücken, besonders an der Steißbeinspitze, Schmerzen, die so heftig seien, daß er weder stehen noch sitzen könne. Seit Pfingsten I9I2 wären diese Beschwerden vcrstärkt. Seit dieser Zeit hätten auch die Durchfälle zugenommen, an denen er, soweit seine Erinnerung reiche, sein ganzes Leben lang schon litte. In den letzten Monaten habe er bis zu zehnmal täglich dünnbreiigen und stets stark mit Blut vermischten Stuhlgang gehabt. Früher seien die Durchfälle weniger häufig, etwa 3-4mal täglich, aufgetreten und auch nicht so regelmäßig und viel weniger stark blutig durchsetzt gewesen. Aber einer Zeit, in der normaler Stuhlgang bestanden, kann Patient sich nicht erinnern. Nach der Operation im Jahre 1908 wäre zunächst eine wesentliche Besserung auch in seinem Allgemeinbefinden eingetreten. Die Durchfälle hätten nachgelassen, für Tage ganz aufgehört, allmählich jedoch sei der Zustand wieder derselbe geworden. Auf die Frage nach Krankheiten in der Familie gibt Patient an, daß ein Bruder und dessen Sohn ebenfalls seit ihrer frühesten Kindheit an denselben blutigen Durchfällen litten.

B ef und: Goertz ist ein mittelgroßer, schmächtig gebauter Mann von blaß gelblicher, an Kachexie erinnernder Gesichtsfarbe, in schlechtem Ernährungszustande (50 kg).

An den inneren Organen lassen sich krankhafte Veränderungen nicht nachwcisen. Ls besteht eine ausgesprochene Schmerzhaftigkeit am Kreuzbein, besonders an der Spitze. Bei der digitalen Untersuchung des Rektums wird ein halbfaustgroßer, zerklüfteter, blumenkohlartiger Tumor an der hinteren. Wand festgestellt. Eine Abgrenzung des Tumors, der etwa $7 \mathrm{~cm}$ oberhalb des Sphincter externus beginnt, nach oben gelingt nicht. Das Darmrohr ist gut durchgängig.

Nach genügender Vorbereitung des Patienten durch Abführmittel, 
Darmspülungen und schließlich Opiumdarreichung, Vornahme der Operation nach Kraske in Äthernarkose am 25. X.'1912.

Durch einen Medianschnitt, der von der Steißbeinspitze über das Kreuzbein weg verläuft, wird das erstere samt dem unteren größeren Anteil des letzerten freigelegt. Die Resektion erfolgt zwischen dem 3. und 4. Kreuzbeinwirbel, unter Durchtrennung der ansitzenden Muskulatur und Bandverbindungen. Beim Abschälen des mit dem Kreuz. bein fest verwachsenen Rektums ergießen sich reichliche Mengen einer schmierigen Flüssigkeit. Von der Operationshöhle aus kann man jetzt eine apfelgroße Resistenz fühlen, die nach oben hin nicht abgrenzbar ist. Das Rektum wird weiter stumpf von seinen Verwachsungen gelöst nach der Prostata und der Blase hin. Diese läßt sich leicht zurückschieben, und an ihr wird das Peritoneum, nachdem es weit hinauf abgelöst ist, durch Nähte fixiert. Die Durchtrennung des Rektums erfolgt halb handbreit oberhalb des Tumors und der hinteren peritonealen Umschlagsfalte im gesunden Gewebe. Nach Abtrennung vom Peritoneum wird das obere Darmende in die Wunde gezogen und durch Nähte mit der Haut vereinigt. Beim Betasten des eben fixierten Darmendes finden sich noch einige Polypen, die umstochen, nach beiden Seiten abgebunden und mit der Schere abgetragen werden. Tamponade mit Vioformgaze, Handtuchverband.

Beim ersten Verbandwechsel zeigt es sich, daß das in die Haut eingenähte Darmende nicht anheilt; die Nähte sind durchschnitten. Das Allgemeinbefinden ist in den ersten Wochen infolge des schweren Eingriffs, der mit starkem Blutverlust verbunden war, so schlecht, $\mathrm{da} B$ der Tod jeden Tag erwartet wurde. Indessen setzte bei aus. gezeichnetem Appetit langsam eine allmähliche Erholung ein. $\mathrm{Zu}-$ gleich füllte sich die durch die Resektion entstandene tiefe Wundhöhle nach und nach mit kräftig wuchernden Granulationen immer mehr aus. So hatte sich das Wundgebiet durch von den Rändern vorschreitende Epithelialisierung um Weihnachten bis auf die Hälfte geschlossen. Das untere Darmende ist in die Granulationsmassen fest eingelagert. Im Laufe des Januar jedoch beginnt sich der $\mathrm{Zu}$ stand $z u$ verschlechtern. An den Sitzknorren sind kleine Dekubitalgeschwüre aufgetreten, die trotz aller Pflege schnell tiefer gehen. Das linke Bein wird stark ödematös und enorm schmerzhaft infolge einer entzündlichen Thrombose des Oberschenkels. Im weiteren Verlauf machte eitrige Abszedierung größere Incisionen nötig, aus denen sich große Mengen eitriger Flüssigkeit täglich entleeren. Viel zu leiden hat Patient unter dem täglich einmal vorgenommenen Verbandwechsel, bei dem es regelmäßig auch stark aus den Gra. nulationen blutet. Der Stuhl ist - das sei ganz besonders hervor. gehoben - auch nach Absetzen des Opiums mittelfest; Durchfälle werden nicht beobachtet. Das Aussehen des außerordentlich ma. geren Patienten, der Mitte Januar $43 \mathrm{~kg}$ wiegt, wird immer elender, 
die Temperatur schwankt um 38 Grad. Ende März ist die Wunde zwar fast völlig geheilt, die Dekubitalgeschwüre dagegen sind bis auf beide Sitzbeinknorren vorgedrungen, so daß der Knochen frei liegt, und auch an beiden frei vortretenden Schulterblättern zeigen sich Dekubituswunden; aus den um Weihnachten angelegten Incisionen anı Oberschenkel eitert es dauernd sehr stark. Bei den heftigen Schmerzen, besonders auch im linken Bein, das in den letzten Wochen nach ciner kurz clauernden Abschwellung wieder ödematös anzuschwellen beginnt, wird jetzt täglich reichlich Opium gegeben. Infolge der enorm stark unterhaltenen Eiterung aus dem Oberschenkel und den unaufhaltsam vorschreitenden Drucknekrosen nehmen die Kräfte jetzt sehr schnell ab. Der Tod erfolgte am 25. IV. I9I3.

Die Sektion wurde wenige Stunden später gemacht. Ich gebe aus dem Protokoll das wesentliche wieder:

Am linken Oberschenkel der bis zum Skelett abgemagerten Leiche finden sich zwei lange, in die Tiefe führende Fisteln, aus denen sich auf Druck ca. I Liter dünnflüssiger Eiter entleert. Auf der Höhe der beiden Darmbeine, besonders an ihren Rändern und an den Spinac, große Dekubitusflächen, in denen teilweise der Knochen frei zutage liegt. Die untere hintere Kreuzbeinfläche ist in einer Ausdchnung von zwei Handtellergröße in eine Eiter sezernierende Wunde verwandelt. Das Steißbein und cin Teil des Kreuzbeins ist durcli Operation entfernt. Hier findet sich eine muldenförmige Wundhöhle, aus der das untere Darmende mit seiner Schleimhaut sich leicht vorwölbt.

Innere Organe.

Außer einigen unwesentlichen Veränderungen findet sich: Braune Atrophie des Herzens, beginnende Endaortitis oberhalb der Klappen, sulzige Umwandlung des noch vorhandenen Fettes, Emphysem beider Lungenoberlappen, $\ddot{O} \mathrm{dem}$ und Hypostasen beider Unterlappen. Vergrößerung und derbe Konsistenz der Milz. Fettige Degeneration und trübe Schwellung beider Nieren, besonders der linken.

Magen nicht vergrößert, ohne Inhalt. Schleimhaut leicht injiziert und stark gewulstet.

Die Dünndarmschleimhaut ist ebenfalls injiziert, stellenweise stark gerötet. Sonst ohne Veränderungen.

Die Schleimhaut des Colons ist mit kleinerbsen- bis haselnußgroßen, durchweg langgestielten traubenförmigen Polypen bedeckt (Fig. o). Besonders reichliche Anhäufungen finden sich unterhalb der Ileocöcalklappe, auch an den Flexuren und nach dem Rektum zu. Auffallend intensive Injizierung zeigt die Schleimhaut unterhalb der Ileocöcalklappe in einer Ausdehnung von etwa zwei Handbreiten. Im retroperitonealen Gebiet längs der Aorta sind die Drüsen vermehrt und zum Teil erheblich vergrößert; sie erreichen Walnußgröße, haben cine frisch rötliche Farbe und weiche Konsistenz. 
Pathologisch-anatomische Diagnose: Polyposis coli et decti. Carcinoma recti.

Der seinerzeit durch Resektion entfernte Tumor ist im Wachstum schon weit vorgeschritten, er hat einen Längen- und Querdurchmesser von ca. $10 \mathrm{~cm}$ eine mittlere Dicke von $2-3 \mathrm{~cm}$. In der Mitte besteht eine dreimarkstückgroße Ulceration mit zerklüfteten wulstig und wallförmig aufgeworfenen Rändern. Die Oberfläche ist durch unzählige kleine linsenförmige Erhebungen ausgebuchtet.

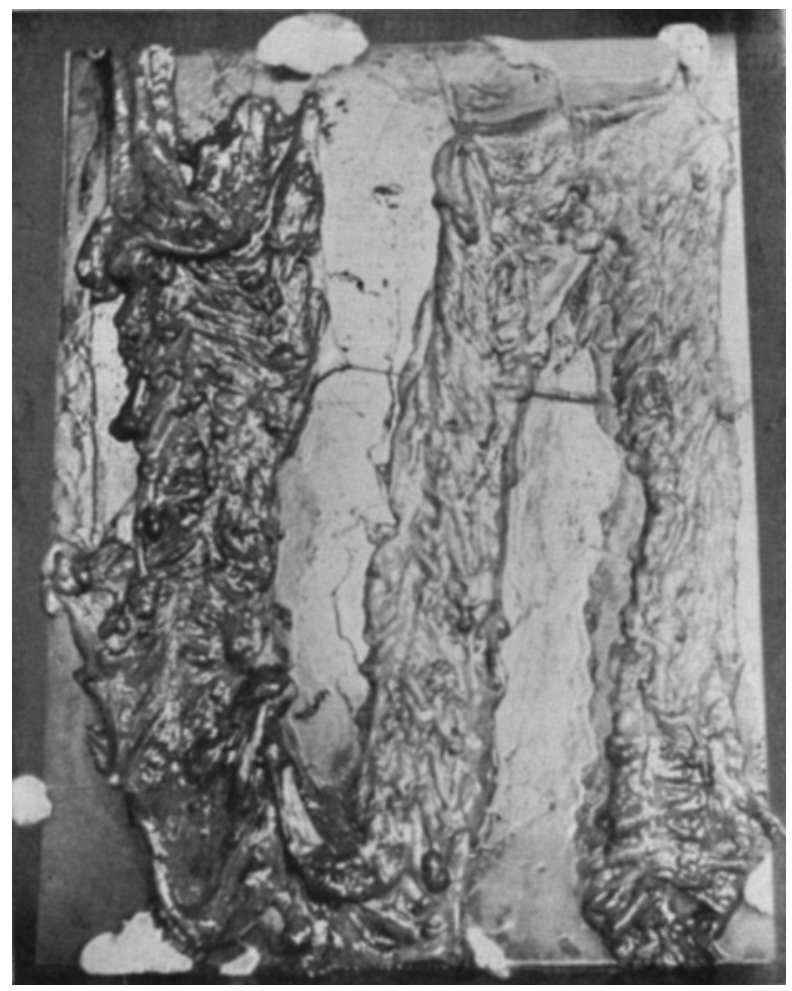

Für die mikroskopische Untersuchung wurde ein Stück aus dem Tumor geschnitten, und zwar von seinem Rande durch das krebsige Gewebe noch in das augenscheinlich gesunde hinein. Die Einbettung erfolgte in Zelloidin und Paraffin.

Auf dem Durchschnitt sieht man vom Rande aus beginnend normales Drüsenepithel. Die Zylinderepithelien sind hier einschichtig. Uberall finden sich Becherzellen. Die Drüsenschläuche liegen gleichförmig nebeneinander. Hier und da sieht man zwischen ihnen und an der tunica propria Zellanhäufungen. 
Die tunica propria zeigt an zwei Stellen starke zellige Infiltrationen. Diese sind noch ausgesprochener und häufiger in der Muscularis. In diese dringen allenthalben schon Carcinomzellen ein, die durch ihre intensivere Kernfärbung sich deutlich aus dem umgebenden Gewebe hervorheben. Die Zellen sind meist zu langen Zügen angeordnet - augenscheinlich folgen sie den Lymphspalten dann aber auch wieder massenhaft zusammengeballt. Verfolgen wir diese Stränge weiter, so liegt bald das typische Bild des Adenocarcinoms vor uns. Ein regelloses Durcheinander von längs und quer getroffenen, dicht nebeneinander liegenden, nur selten durch zartes Bindegewebe getrennten Drüsenschläuchen. Ihre Lumina weisen die mannigfaltigsten Formen und Weiten auf, zum Teil sind sie deutlich offen, zum Teil so dicht aneinander gedrängt, daß sie massiv erscheinen. An manchen Stellen sieht man sie ganz ausgefüllt mit kernreichen Drüsenepithelien. Die Zylinderepithelien haben hier und da einen regelmäßigen einschichtigen Bau, meistens sind sic jedoch mehrschichtig mit ungleich hoch angeordneten Kernen.

Dic zum Teil schr starken Bindegewebsstränge, welche die Carcinommassen viclfach durchzichen, weisen zahlreiche Zellanhäufungen auf. Durch zellige Infiltrationen erscheint der Übergang von Tumor zu normalem Gewebe scharf begrenzt. Bei stärkerer Vergrößerung sieht man indessen, wie überall langgestreckte Drüsenschläuche und einzelne, meist dicht aneinander gedrängte Zellreihen sich in das normale Gewebe vorschieben.

Dic Polypen zeigen im mikroskopischen Bilde sämtlich den typischen Bau der Schleimhautpolypen: vermehrte, verlängerte und verzweigtc Drüsenschläuche mit einschichtigem gleichmäßigen $\mathrm{Zy}$. linderepithel.

Die untersuchten retroperitonealen Lymphdrüsen sind mäßig infiltriert.

F all 2. Über den eingangs erwähnten Bruder des verstorbenen Patienten findet sich in den Krankenjournalen folgendes:

Fritz G., 26 Jahre, Arbeiter. Salzdetfurt 29. VII. bis I5. VIII. yol.

Diagnose: Papillomata recti.

A namnese: Patient leidet angeblich schon seit vielen Jahren an Hämorrhoiden. Wurde deswegen einige Male im hiesigen St. Bernwardkrankenhaus operiert. Die Hämorrhoiden seien aber stets wiedergekommen und träten stets beim Stuhlgang aus. Seit gestern könnten sie nicht wicder zurückgebracht werden. Früher will $\mathrm{Pa}$ tient nic ernstlich krank gewesen sein.

S t a tu s: Mittelgroßer, mittelkräftig gebauter Mann von blassem Aussehen und mäßiger Ernährung.

Innere Organe ohne Befund. In der Rima ani liegt ein etwa fausigroßes Pakct von einzelnen größeren und kleineren Polypen, 
die der zum Teil prolabierten Rektumschleimhaut gestielt, selten mit breiterer Basis aufsitzen. Sie sind sehr zerreißlich und bluten leicht; lassen sich ohne Mühe reponieren, da der After sehr weit ist.

Es wurden Abführmittel und Sitzbäder verordnet.

4. VIII. In Äthernarkose wurden die einzelnen Papillome nach vorheriger Unterbindung bzw. bei breiterer Basis Umstechung an der Schleimhaut abgetragen. Ziemlich starke Blutung. Auf der Schleimhaut sind noch zahlreiche Knötchen - beginnende Papillome - die sich nicht entfernen lassen.

15. VIII. Beschwerdefrei entlassen. Gewicht $661 / 2 \mathrm{~kg}$.

Gelegentlich seiner Krankenbesuche bei dem Bruder wurde dieser Fritz G. eingehend untersucht.

Anamnestisch machte er zunächst die Angabe, daß nach der IgoI ausgeführten Operation eine kurzdauernde Besserung eingetreten sei. Allmählich habe sich aber der alte Zustand mit blutigen Durchfällen und Schmerzen wieder hergestellt. Jedoch seit Pfingsten 19I3 etwa hätten die Durchfälle angefangen seltener zu werden, jetzt habe er schon monatelang keine Beschwerden mehr und durchaus regelmäßigen Stuhlgang ohne Blutbeimischung.

Er ist ein blaß, leicht kränklich aussehender Mann in mittlerem Ernährungszustand. - Die inneren Organe sind gesund.

Die rektale Untersuchung ergibt das. Vorhandensein von kleinerbsengroßen und meist lang gestielten Polypen an der hinteren Rektumwand, etwa $7 \mathrm{~cm}$ oberhalb des Sphincter externus beginnend. Kein Carcinom.

F all 3. Im wesentlichen derselbe Befund findet sich bei der Untersuchung des (einzigen) 5jährigen Sohnes. Auch hier sitzen der hinteren Rektumwand unzählige Polypen auf, zum Teil gestielt, zum Teil aber auch nur als kleine Vorsprünge fühlbar.

In einem auffällig hohen Prozentsatz tritt bei der Polyposis Carcinombildung auf, eine Tatsache, auf die man schon früh aufmerksam wurde und auf die immer wieder hingewiesen wird. In der Döringschen Zusammenstellung von Polyposisfällen gehen 24 unter $50=46$ Proz. mit carcinomatöser Entartung einher. Bei Versé sind die entsprechenden Zahlen $27 \mathrm{zu} 57$. Nach Zufügung der von mir aus der Literatur zusammengestellten und der hier beobachteten Fälle ergibt sich ein Verhältnis von $39 \mathrm{zu}$ 8o, also ca. 50 Proz. Carcinomdegeneration bei Polyposis.

Die Krankheit bevorzugt in ausgesprochener Weise das männliche Geschlecht. So findet Döring unter 42 Erkrankten 25 Männer und 17 Frauen, also ein Verhältnis von 100 zu 67, ähnlich Wechselmann. Aus der Verséschen Statistik läßt 
sich der Prozentsatz mit $100 \mathrm{zu} 60$ berechnen. In meiner $\mathrm{Zu}$ sammenstellung entfallen unter 22 Erkrankungen auf das männliche Geschlecht I2 und auf das weibliche 9. Alles in allem ist bei 74 Gesamterkrankungen die männliche Beteiligung mit 44 gegenüber 30 des weiblichen Geschlechts, also mit IOo zu 68 immerhin sehr erheblich. Noch größer ist der Unterschied in den entsprechenden Zahlen, was die Verteilung der Polyposis mit Carcinomkombination betrifft.

Hier ist das Prozentverhältnis bei Döring I 4 zu 7, bei Versé $I 7 \mathrm{zu} \mathrm{8,} \mathrm{in} \mathrm{meiner} \mathrm{Zusammenstellung} 6 \mathrm{zu} \mathrm{4.} \mathrm{Im} \mathrm{ganzen}$ sucht also die Polyposis mit Carcinom das männliche Geschlecht doppelt so häufig heim wie das weibliche.

Im Gegensatz zu den übrigen Tumoren befällt das Leiden vorzugsweise das jugendliche und mittlere Alter. Der Beginn fällt in eine in den einzelnen Fällen häufig wahrscheinlich um viele Jahre, ja Jahrzehnte zurückliegende Zeit. Ganz besonders zeigt sich das bei unsern drei Kranken, die bei einem Alter von 28, 38 und 5 Jahren alle schon von erster Kindheit an die klinischen Symptome der Polyposis - blutige Durchfälle - aufweisen. Über die Verteilung der Krankheitsfälle auf die einzelnen Jahrzehnte mögen folgende Zusammenstellungen ein Bild geben:

\begin{tabular}{l||r|r|r}
\hline & Versé & $\begin{array}{c}\text { Eigene } \\
\text { Zusammen- } \\
\text { stellung }\end{array}$ & Gesamt \\
\hline \hline 1-10 Jahr & 4 & 3 & 7 \\
10-20 " & 13 & 3 & 16 \\
$20-30, "$ & 8 & 4 & 12 \\
$30-40$, & 13 & 5 & 18 \\
$40-50$, & 4 & 3 & 7 \\
$50-60$, & 2 & - & 2 \\
$60-70$, & 1 & 3 & 4 \\
\hline & 45 & 21 & 66
\end{tabular}

Nach Versé erkranken also bis zum 4o. Lebensjahre an Polyposis 38 gegen 7 jenseits des 4o. Lebensjahres. Bei den von mir zusammengestellten Fällen kommen I 5 auf die 4 ersten Jahrzehnte und 3 auf den Rest. Danach entfallen ca. 82 Proz. aller Erkrankungen auf die Zeit bis zum 40. Lebensjahre und nur I 8 auf die übrigen. Die Fälle mit Carcinombildung verteilen sich folgendermaßen: 


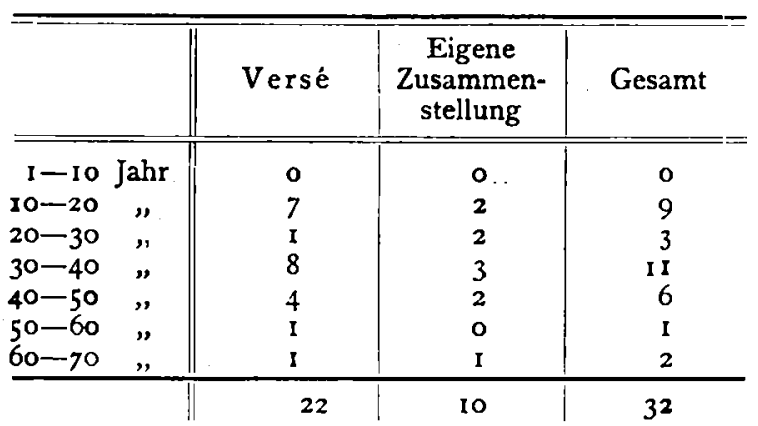

Das ergibt ein Prozentverhältnis von 100 Erkrankunger innerhalb der ersten 40 Jahre gegen 40 der übrigen.

Schon früh wurde die Beobachtung gemacht, daß das Leiden häufig bei mehreren Mitgliedern derselben Familie auftritt. Leider fehlen in den meisten Berichten Angaben über die so wichtige Frage, da uns häufig nur Sektionsbefunde zur Verfügung stehen und die Fälle hauptsächlich in histogenetischer Hinsicht bearbeitet sind. So wird man die von Wechselmann mit 50 Proz. angenommene Heredität nicht als $z u$ hoch gegriffen bezeichnen. Eine zahlenmäßige sichere Feststellung läßt sich eben bei der geringen Zahl genügend untersuchter Fälle nicht machen. Die folgenden familiären Fälle habe ich aus der Tabelle Döring bzw. Versés zusammengestellt mit Angabe des Beobachters, des Alters und Geschlechts der Patienten.

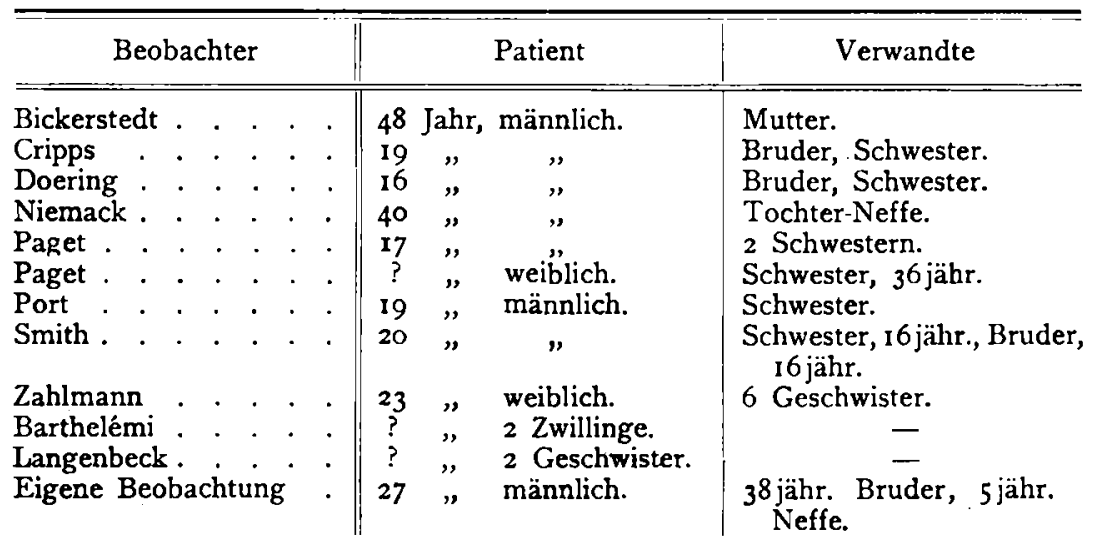

Die Fälle von Barthélemi und Langenbeck sind in der Arbeit von Wechselman n ,Polyp und Carcinom im Magen- 
Darmkanal" ohne nähere Einzelheiten angegeben; in der Literatur habe ich sie nicht auffinden können. Auch hier ist wieder auffallend die hohe Beteiligung des männlichen Geschlechts an der krebsigen Entartung: Es sind erkrankt 8 Männer und nur 2 Frauen. Die Carcinombildung fällt mit 2 Ausnahmen in das 2. und 3. Lebensjahrzehnt.

Was den Sitz der Polyposis betrifft, so finden wir, daß der Dickdarm am meisten bevorzugt ist. Für die Frage nach der Ätiologie dürfte es vielleicht nicht ganz ohne Interesse sein, daß die Polypen an den Flexuren und im Rektum oft, ja fast immer gehäuft auftreten. Im Dünndarm kommt die Erkrankung so gut wie gar nicht vor, ebenso wenig im Magen. Zur besseren Orientierung über die Verteilung auf die verschiedenen Darmabschnitte dient eine von W echselmann nach der Verséschen Statistik gemachte Zusammenstellung, der ich unsere Fälle hinzufüge.

\begin{tabular}{|c|c|c|c|}
\hline & $\begin{array}{c}\text { Wechsel. } \\
\text { mann }\end{array}$ & $\begin{array}{c}\text { Eigene } \\
\text { Beobachtung }\end{array}$ & Gesamt \\
\hline I. Cöcum . & 3 & 3 & 6 \\
\hline 2. Colon & 4 & 5 & 9 \\
\hline 3. Rektum . . & 16 & 7 & 23 \\
\hline 4. Cöcum + Colon. & 1 & o & I \\
\hline 5. Rektum + Colon . . . . . & 13 & 8 & 21 \\
\hline 6. Cöcum + Colon + Rektum . & 7 & 3 & 10 \\
\hline 7. Ileum :. . . . . . & 2 & - & 2 \\
\hline 8. Ileum + Colon + Rektum . & 2 & - & 2 \\
\hline 9. Duodeum + Colon + Rektum . & 1 & - & I \\
\hline Io. Magen + Jejunum + Ileum . . & 1 & - & I \\
\hline II. Magen-Cöcum-Colon-Rektum & 1 & - & I \\
\hline 12. Magen - Duodenum - Colon - & I & - & I \\
\hline 13. Ges. Tractus außer Jejunum . . & I & - & I \\
\hline 14. Ges. Tractus. . . . . . . . & 2 & - & 3 \\
\hline
\end{tabular}

Bemerkenswert ist, daß das Rektum nach dieser Zusammenstellung in 63 unter 76 Erkrankungen mitbeteiligt ist. Das ergibt ein Prozentverhältnis von über 83. Nächst dem Mastdarm findet sich das Colon mit 60 Proz. am häufigsten erkrankt. In Wirklichkeit muß der Wert bedeutend höher angenommen werden, da nach den bisherigen Obduktionsbefunden nur ganz vereinzelte Fälle ohne Beteiligung des Colons - wenigstens seines unteren Teiles - vorliegen. In nur 4 Fällen ist der Dick- 
darm frei von Polypen. Auffallenderweise wird nur einmal von einer Polyposis des Dünndarms berichtet. Man kann also sagen, daß die Polyposis eine Erkrankung der Dickdarmschleimhaut darstellt.

Über den Anteil, welchen die verschiedenen Darmabschnitte an der Carcinombildung haben, möge folgende Tabelle Aufschluß geben:

\begin{tabular}{|c|c|c|c|}
\hline & Versé & $\begin{array}{c}\text { Eigene } \\
\text { Zusammen- } \\
\text { stellung }\end{array}$ & Gesamt \\
\hline $\begin{array}{l}\text { Rektum } \\
\text { Colon . } \\
\text { Colon + Rek- } \\
\quad \text { tum. } \\
\text { Ileum. . }\end{array}$ & $\begin{array}{r}16 \\
5 \\
2 \\
1\end{array}$ & $\begin{array}{r}7 \\
1 \\
3 \\
-\end{array}$ & $\begin{array}{r}23 \\
6 \\
5 \\
I\end{array}$ \\
\hline & 24 & II & 35 \\
\hline
\end{tabular}

Mit nur einer Ausnahme (Fall von Thorel), die zudem nach Versé mit unbedingter Sicherheit nicht zur Polyposis gerechnet werden darf, ist also die Dickdarmschleimhaut auch Sitz der Carcinombildung, und zwar ist mit 75 Proz. das Rektum auch hier hauptbeteiligt.

In einigen Fällen wird zwar von einem rapiden Verlauf der Polyposiserkrankung berichtet. Es scheint aber doch, als ob sie häufiger noch sich über viele Jahre hinzöge. Dabei ist zu beachten, daß jedenfalls viele leichtere Erkrankungen, die ohne auffällige klinische Erscheinungen verlaufen, nie zur Beobach. tung kommen. Angaben, daß die Krankheit seit frühester Kindheit besteht, finden sich häufiger. Ich möchte unsere drei hier béobachteten Fälle als in dieser Beziehung charakteristisch anführen. Bei dem 38 jährigen Fritz G. wurde vor I2 Jahren eine Polypenoperation vorgenommen und dabei das Vorhandensein von multiplen Polypen diagnostiziert. Schon viele Jahre vor dieser Operation hatte Patient im Bernward-Krankenhaus 1894 sich der gleichen Operation unterzogen. Die Krankheit ist also schon vor 19 Jahren festgestellt worden. In Wirklichkeit besteht sie seit der frühesten Kindheit, das können wir mit Sicherheit aus den k̇linischen Erscheinungen, nämlich den häufigen blutbeigemischten Durchfällen schließen. Genau so verhält es sich mit dem 
27 jährigen, jetzt verstorbenen Bruder. Auch hier traten blutige Durchfälle schon in der Kindheit auf. I 908 kam er wegen Prolapsbeschwerden zum ersten Male zur Beobachtung, und bei der Operation wurde die Diagnose Polyposis recti gestellt. Und auch das 5 jährige Kind $G$. leidet, wie die Mutter angibt, ,seit der Geburt" an Durchfällen, die in den letzten Jahren häufiger Blutbeimischungen zeigen. Bei der Unsicherheit der anamnestischen Angaben, die zum großen Teil in der mangelhaften Selbstbeobachtung begründet sind, hat eine Übersicht über die nach ihren Erscheinungen bemessene Dauer der einzelnen Fälle natürlich nur bedingten Wert. Sicherer AufschluB über die wirkliche Dauer ist kaum zu erwarten. In einem Falle dauert die Krankhcit nur 3 Wochen, je I mal 3 und 7 Monate; 6 mal läßt sich das Leiden auf die ersten Lebensjahre zurück verfolgen. Bei etwa 40 Proz. der Erkrankungen überschreitet die Dauer ein Jahrzehnt. Wie schon ausgeführt wurde, ist dieser Prozentsatz in Wirklichkeit bedeutend höher anzunehmen.

Die Frage nach der Entstehung der Polypen ist noch offen und Gegenstand lebhafter Erörterungen, die jedenfalls in absehbarer Zeit noch nicht zu einer Klärung der Ansichten führen werden. Es sind im wesentlichen zwei Theorien über die Histogenese aufgestellt, von denen die eine entzündliche Reize für die Entstehung verantwortlich macht unter Annahme ev. einer angeborenen Disposition der Darmschleimhaut. Versé redet von einer „kongenitalen Disposition der Epithelzelle zu stärkerer Proliferation“; er erklärt die Entstehung der Polypen so, daß sich die Schleimhaut faltet, die vorspringenden Stellen gereizt werden, deshalb schärfer zu wuchern beginnen und intensiver vorspringen. Nach Wechselmann, ,bringen katarrhalische Affektionen keine Polypen hervor, sondern Schleimhautwucherungen ganz anderer Art, die in das Gebiet der Hyperplasien gehören". So ist denn auch die allgemein verbreitete Anschauung die, daß es sich bei dem Polypen um angeborene, aus Entwicklungsstörungen entstandene Bildungen, um Zellausschaltungen handelt. Für die Richtigkeit dieser ausgebauten modifizierten Cohnheimschen Theorie scheint das familiäre Auftreten der Polyposis zu sprechen, ferner auch die Tatsache, daB in auffälliger Weise das jugendliche Alter von der Erkrankung be- 
troffen ist. Bei Betrachtung z. B. unserer 3 Fälle, bei denen das Leiden doch ganz augenscheinlich seit erster Kindheit besteht, Vater, Sohn und Onkel, unter ganz denselben Erscheinungen und bei gleich lokalisierten Untersuchungsbefunden erkrankt sind; wird man mit der Annahme lediglich einer angeborenen Disposition der Schleimhaut zu der in jedem Falle ein längerer andauernder Reiz treten müßte, keine recht befriedigende Erklärung finden können. Auch Hauser hält die Polyposis ,für eine primäre Erkrankung oder Entartung des Darmepithels, wobei die Entzündung nur begünstigend für das weitere Wachstum wirkt". Wie die Zellausschaltung zustande kommt, ob sie - wie Wechselmann annimmt - sozusagen mehr lokaler Natur ist und durch Windungen und Drehungen des Darmes entsteht, oder nach Ribberts phylogenetischer Erklärung durch Keimüberfluß, durch Überschuß an ursprünglicher Darmschleimhaut bedingt ist, dürfte kaum je zu entscheiden sein.

Aus den Polypen nun entwickelt sich das Carcinom. Das steht fest. Scharf umstritten aber ist die Frage, wie der Krebs sich bildet. Hier stehen sich zwei Anschauungen gegenüber. Die von $\mathrm{Ha}$ user vertretene hat allgemeinere Anerkennung gefunden, daß nämlich die Epithelzellen sich primär umwandeln und daß der Polyp als ganzes Gebilde krebsig entartet. Für diese Auffassung tritt u.a. Versé auf Grund einer Reihe von mikroskopischen Untersuchungsbefunden ein. In neuester Zeit beobachtete auch Oseki unzweifelhafte Übergangsbilder der adeno. matösen Polypen zu den Carcinomzellensträngen. Das Bindegewebe spielt bei der Krebsentstehung und Wachstum gar keine Rolle. Es verhält sich passiv oder reagiert nur entzündlich auf die Epithelwucherung. „Die Drüsen wuchern aktiv ohne Mithilfe des Bindegewebes in die Tiefe." Im Gegensatz zu dieser Entstehungserklärung verteidigt $\mathrm{R}$ ib be $\mathrm{r} t$ jetzt in seinem neuesten Werke „Das Carcinom des Menschen" warm seine Theorie, nach welcher der Anlaß der Entstehung des Krebses im Bindegewebe zu suchen ist. Die Ribbertsche Theorie ist in dem Schlußsatze seines eben angeführten Werkes zusammengefaßt: „Das Carcinom entsteht auf Grund einer durch Epithelprodukte bewirkten, die Differenzierung des Epithels vermindernden und sein Tiefenwachstum auslösenden subepithelialen Entzündung“. Weiter ver- 
wirft Ribbert entschieden die Auffassung von einer allgemeinen krebsigen Degeneration der Polypen und betont immer wieder, daß der Krebs am oder aus einem Polypen entsteht, niemals aber ein Polyp sich in ein Carcinom umwandelt oder in allen seinen Teilen gleichzeitig ein carcinomatöses Wachstum eingeht. Es scheint, als ob die Beurteilung der mikroskopischen Befunde seitens der einzelnen Forscher durch die von ihnen vertretenen Theorien nicht ganz unbeeinflußt blieben, oder daß doch jedenfalls der Deutung und Auslegung in den einzelnen Fällen ein großer Spielraum bliebe. So kommen z. B. Ribbert und Versé bei ihren Untersuchungen beginnender polypöser Carcinome häufig genau zu den entgegengesetzten Resultaten. Es findet nämlich der eine da eine scharfe Grenze zwischen Carcinom und Polyp, wo von dem andern mit derselben Sicherheit festgestellt wird, daß eine, immer weiter gehende Umwandlung des ursprünglich normalen Epithels bis zum Carcinom stattfinde, wobei zwischen den einzelnen Stadien fließende Übergänge nachzuweisen sind". Ich möchte hier eine Äußerung von Lubarsch über die Streitfrage der Histogenese anführen, die sich in einem Aufsatz über die Grenzen der pathologischen Anatomie und Histologie findet (Jahreskurse für ärztliche Fortbildung I9I3, Januar). Danach ist die Frage „ob die im Gebiet vieler Krebse subepitheliale entzündliche Zellausscheidung und Wucherung primär oder sekundär ist, d.h. ob durch diese Zellansammlung die Epithelwucherung erst ermöglicht und ausgelöst wird, oder ob die Entzündung erst Folge der Epithelwucherung ist" durch histologische Untersuchungen nicht sicher zu entscheiden. Nachdrücklich wendet sich Lubarsch gegen die Auffassung, daß man z. B. sehen kann, wie sich der Krebs aus den Drüsenepithelien entwickelt. Das sei deswegen unmöglich, weil stets gewisse fertige Stadien vorlägen.

In ihrem Beginn macht die Polyposis sehr häufig ja fast regelmäßig die Erscheinung eines Dickdarmkatarrhs. Es kommt zu Durchfällen, die früher oder später fast stets Beimischungen von Blut und Schleim zeigen. Die Diarrhöen und die selten ausbleibenden schmerzhaften Tenesmen führen $\mathrm{zu}$ allmählicher Abzehrung, ja zum Tode. Auch von starken Blutungen wird berichtet, die in kurzer Zeit tödlich endeten. Bei Patienten, die 
an dauernden Durchfällen leiden, kommt es leicht zum Vorfall von Polypen. Die Folge sind dann meist schmerzhafte Einklemmungserscheinungen, unter Umständen sogar das Leben direkt gefährdende Blutungen. Auch Rektalprolapse mit ihren Folgeerscheinungen sind beobachtet worden. Auch bei unseren Fällen ist die Mehrzahl der geschilderten Symptome in - ich möchte sagen - klassischer Weise vereinigt. Das Leiden beginnt mit Durchfällen, zu denen bald Blutbeimischungen treten. Die Patienten fühlen sich matt. Ihr Aussehen ist bla $B$ und elend. Später treten bei den beiden Brüdern Polypen aus. Schmerzhafte Einklemmung, starke Blutung vervollständigen diesen typischen Symptomenkomplex der Polyposis.

Die exakte Diagnose ist natürlich nur durch digitale Untersuchung, besser noch mit Hilfe der Romanoskopie zu stellen. Da das Rektum fast stets an dem Prozesse mitbeteiligt ist, so wird kaum ein romanoskopisch untersuchter Fall unerkannt bleiben. In seltenen Fällen hat das Abgehen von Darmpolypen mit dem Stuhl oder das Vordrängen aus dem Rektum auf das Leiden aufmerksam gemacht. Im übrigen sollte man bei dem Bilde, das die oben geschilderten Symptome, besonders die blutig. schleimigen Durchfälle darbieten, stets an die Möglichkeit einer Polyposiserkrankung denken.

Leider ist damit für den Kranken nicht viel gewonnen, denn von der Therapie haben wir nach den bisherigen Ergebnissen und Erfahrungen wenig oder fast nichts zu erwarten. Interne Behandlung, die in Spülungen mit adstringierenden Mitteln bestand, hat in einigen Fällen nur kurz andauernde Besserung erzielt. So hatte Funkenstein vorübergehenden Erfolg mit Chinineingießungen. Döring sah Besserung nach Tanninspülung. Ohne Wirkung war die Spülung bei einem Falle von Praag. Bablers Patient, dem vom eingenähten Wurmfortsatz aus der Dickdarm gespült wurde, starb 8 Tage nach der Operation.

Wer auf dem Standpunkte steht, daß die Polyposis ein kongenital bedingtes Leiden ist, wird auf jede interne Behandlung von vornherein verzichten.

Von Operationsmethoden erscheint am naheliegendsten wohl die Abschabung oder Abtragung der Polypen - soweit sie er- 
reichbar sind. Von den Io bekannten Fällen, die so behandelt wurden, sind 3 gestorben, 3 angeblich gebessert; einen Fall (von Dun) gibt Versé als geheilt an. Bei 2 Patienten traten Rezidive auf, die wegen carcinomatöser Entartung nach 6 bzw. 4 Jahren die Resektion des Rektum nötig machten.

Merkwürdigerweise ist der von uns beobachtete Fritz G., nachdem wiederholten Abtragungen von Polypen merkliche Besserung nicht gefolgt war, seit Pfingsten I9I2 frei von allen Beschwerden. Immerhin wird man - Dauerresultate fehlen leider völlig - bei Betrachtung der bisherigen Ergebnisse feststellen müssen, daß wohl eine kürzer oder länger anhaltende Besserung, niemals aber Heilung von der Abtragung zu erwarten ist. Bei der Ausdehnung des polypösen Prozesses können alle Polypen durch Abtragung eben niemals entfernt werden.

Die Überlegung, daß die Polyposis durch den Reiz der Kotmassen hervorgerufen oder doch verschlimmert würde, legte den Gedanken an eine Ausschaltung und damit Schonung des erkrankten Darmteiles nahe. Diesen Zweck hat man in 8 Fällen durch Anlegung eines künstlichen Afters zu erreichen gesucht, und zwar $6 \mathrm{mal}$ an der typischen Stelle der Flexur. In einem Falle (Margarini) wurde ein cöcaler After angelegt und einmal die totale Ausschaltung des Colons durch axiale Einpflanzung des untersten Ileums in die Flexur bewirkt. In diesen Fällen handelte es sich 5 mal um eine Polyposis mit Carcinombildung und 3 mal lag cine unkomplizierte Polyposis vor. Die Resultate der Operationen sind wenig ermutigend: 3 Todesfälle (darunter der Fall von Döring; Palliativoperation bei inoperablem Carcinom), eine Operation versagte, je einmal keine und nur anfängliche Besserung, eine mit unbekanntem Ausgang. Esser berichtet von einer Besserung der Darmfunktionen. $\mathrm{Ob}$ dieses Resultat aber von Dauer bleiben wird, erscheint nach den vorliegenden Ergebnissen zum mindesten zweifelhaft.

Nicht viel besser sind die Erfolge der Exstirpation des Rektums, welche in den 6 Fällen bei carcinomatöser Entartung und 2 mal bei reiner Polyposis vorgenommen wurde. Von den 4 bald nach der Operation gestorbenen Patienten erlagen 2 einer Bronchopneumonie; ein Resultat ist unbekannt. Rotter be- 
richtet von einer Kranken, die 21/2 Jahre nach der Operation an Carcinometastasen im Becken starb. An der Amputationsstelle des Rektums fand man bei ihr keine Spur von Carcinom mehr; auch bei dem hier operierten und 6 Monate nach der Operation gestorbenen W. G. fanden wir im Rektum nichts carcinomverdächtiges. Bei unkomplizierter Polyposis des Rektums erzielte Brentano durch die Kraskesche Operation Besserung, Steinthal zurzeit noch bestehende Heilung. Lindner resezierte das Rektum und einen großen Teil vom Colon descendens. Dies ist der einzige Fall, der 4 Jahre nach der Operation bei der Nachuntersuchung noch rezidivfrei gefunden wurde. Von sonstigen Eingriffen sind einige Male Darmresektionen ausgeführt, durchweg wegen Invaginationserscheinungen, und zwar 3 mal ileocöcal mit zwei Heilungen und einem tödlichen Ausgange an Peritonitis. Karajan resezierte den Darm an fünf Stellen und erzielte Heilung. Denselben günstigen Ausgang hatte eine Resektion von Riederer am Colon transversum. Auch hier wird man mit einem abschließenden Urteil bei der geringen Zahl von Beobachtungen und bei dem Fehlen von Nachuntersuchungen zurückhalten müssen. Prognostisch am günstigsten liegen auch diese letzten Fälle, in denen die Resektion wegen Ileus gemacht wurde. Die Indikation zu diesem Eingriff ist die bekannte. Die Diagnose der Polyposis wird dabei wohl erst stets nach der Eröffnung des Darmes gestellt werden. An eine Amputation des Rektums sollte man nur bei Polyposis mit carcinomatöser Entartung herangehen. Denn da sich immer wieder zeigt, daß die Krankheit fast niemals auf das Rektum beschränkt ist, eine völlige Exstirpation des erkrankten Darmteiles also ausgeschlossen erscheint, so wird durch den schweren Eingriff nur das Leben des Patienten gefährdet oder doch seine Lebenskraft unnötigerweise geschwächt. An diesem Urteil können auch die beiden durch Resektion erzielten, zurzeit noch bestehenden Heilungserfolge nichts ändern. Die Therapie muß eine palliative sein. Man soll, wenn einmal die Diagnose auf Polyposis recti gestellt ist, den Patienten in gewissen Zeiträumen regelmäßig untersuchen und dann unverzüglich die Resektion des Mastdarmes vornehmen, sobald sich carcinomatöse Entartung zeigt. Im übrigen ist bei dem starken Kräfteverlust infolge der fast nie 
fehlenden starken Durchfälle und Blutverluste das Hauptgewicht auf eine kräftige Ernährungsweise zu legen.

Über die Prognose, die in den Ausführungen der Therapie schon berührt wurde, mögen folgende Zahlen einen kurzen Überblick geben: Unter 78 Fällen starben 53, davon 32 an Carcinom, 7 Ausgänge sind unbekannt, I 3 werden als geheilt bzw. als gebesscrt angegeben, 5 als noch lebend. Diese Zahlenverhältnisse können natürlich keinen Anspruch auf absolute Genauigkeit machen. So wissen wir z. B. gar nichts von dem späteren Schicksal der zurzeit der betreffenden Veröffentlichung noch als lebend geheilt oder gebessert angegebenen Patienten. Es müßten, um ein einigermaßen klares Bild zu bekommen, möglichst viele Fälle nachuntersucht werden; dann wird sich wahrscheinlich herausstellen, daß unter den günstigen Resultaten nur wenige Dauererfolge geblieben sind. Es handelt sich also bei der Polyposis intestini um eines der traurigen und fast gänzlich hoffnungslosen Leiden der Menschheit.

Zum Schluß möchte ich Herrn Medizinalrat Dr. Becker, meinem hochverehrten Lehrer, für dic Überlassung der Arbeit und das unterstützende Interesse, das er an ihr genommen hat, meinen herzlichsten Dank aussprechen.

\section{Literaturverzeichnis.}

I. A]bu, A., Benigne und maligne Polypen der Flexura sigmoidea und der Ampulla recti. Berliner klin. Wochenschr. I9I2, Nr. 39.

2. $\mathrm{B}$ abler, $\mathrm{Niebr} \ddot{\mathrm{g} g \mathrm{c}}, \mathrm{F}$ is $\mathrm{Ch}$, Carcinomatous polyposis of the colon. Journal of the med. assoc, Bd. VII, Nr. i6. Ref. Zentralbl. f. Chir. 1909, Nr. 39.

3. B r entano, Polyposis recti et intestini crassi. Zentralbl. f. Chir. Igog, Nr. 15.

4. Charles A. Norton. The Lancet 1895 , p. 1245.

5. Döring, Die Polyposis intestini und ihre Beziehung zur carcinomatösen Degeneration. Arch. f. klin. Chir. 1907, Bd. 83 .

6. Ess er. Deutsch. Arch. f. klin. Med. 1908, Bd. 93, H. 5, 6.

7. Helferich-Kaselowsky, Ein Fall von Polyposis recti. Inaug.Diss. Kase low k $\mathrm{k}$.

8. Küt tner. Berliner klin. Wochenschr. I908, Nr. II.

9. Derselbe, Fall von typ. Polyposis recti et intestini crassi mit Übergang in Carcinom. Allgem. med. Zentralzeitung 1908, Nr. 6, S. 80. 
578 THORBECKE, Über das familiäre Auftreten von Darmpolypen.

10. Oseki, Polyposis adenonatosa mit Carcinoma recti. Deutsche Zeitschr. f. Chir. 1912, Bd. I18, H. 5-6.

I1. Pra ag, Polyposis intestini. Deutsche med. Wochenschr. 1909, Referat.

12. Qućnu u. Landel, Cancers du rectum et de l'anse sigmoide avec polyposi du gros intestini. Revue de chir. 1899.

13. Ribbert, Darmpolyp und Carcinom. Frankfurter Zeitschr. f. Chir. 1908, Bd. 2.

14. Derselbe, Das Carcinom des Menschen. Bonn I9II.

15. Robson-Knaggs. The Lancet 1905 .

16. Schneider, Polyposis intestini beim Kinde. Arch. f. Kinderheilk., Bd. 53, H. 4-6. Ref. Deutsche med. Wochenschr. 1911.

17. St e inthal, Polyposis recti. Deutsche med. Wochenschr. Igro.

18. Versé, Über die Entstehung, den Bau und das Wachstum der Polypen, Adenome und Carcinome des Magen-Darmkanals. Arbeiten aus dem path. Institut zu Lcipzig 1908, Bd. I.

19. Wechselmann, Polyp und Carcinom im Magen-Darmkanal. Beitr. z. klin. Chir. r9ı, Bd. 70. 